\title{
Subcutaneous emphysema: ultrasound barrier
}

\author{
Andrew Verniquet, MD · Rafid Kakel, MD
}

Received: 8 November 2010/Accepted: 18 November 2010/Published online: 4 December 2010

(C) Canadian Anesthesiologists' Society 2010

\section{To the Editor,}

We read with interest the correspondence from Dr. Neustein, "mandating ultrasound usage for internal jugular vein cannulation". ${ }^{1}$ In their reply, Drs. Ayoub and Denault stated that there are limitations to its use. We recently encountered one such limitation.

An elderly patient presented for emergency laparotomy with massive pneumoperitoneum and subcutaneous emphysema of the neck following a colonoscopy. Both sides of the patient's neck were scanned using an L25 6-12 MHz linear transducer with the intention of inserting a central venous catheter via the internal jugular vein. However, no structures could be identified deep to the external jugular vein (Fig. 1). Sometimes, with pressure and patience, it is possible to move the air away from the transducer in order to create a window to view the deeper structures. However, the maneuver was not effective in this case. Thirteen days later, the subcutaneous emphysema had largely resolved (Fig. 2).

Ultrasound loses some of its energy when it travels through a medium (attenuation). At an interface between two media with different acoustic impedances, some of the ultrasound is reflected while the rest passes through the medium. Acoustic impedance (the tendency of the medium to resist the passage of ultrasound) is defined as the product of the density of the medium and the propagation speed of ultrasound in that medium. The units of acoustic impedance, i.e., rayls, are measured in $\mathrm{kg} \cdot \mathrm{m}^{-2} \cdot \mathrm{sec}^{-1}$. Only the ultrasound returning to the transducer is detected. The percentage of ultrasound reflected is defined by the

A. Verniquet, MD $(\bowtie) \cdot$ R. Kakel, MD

James Paton Memorial Hospital - Central Health, Gander, NF, Canada

e-mail: andrewverniquet@hotmail.com

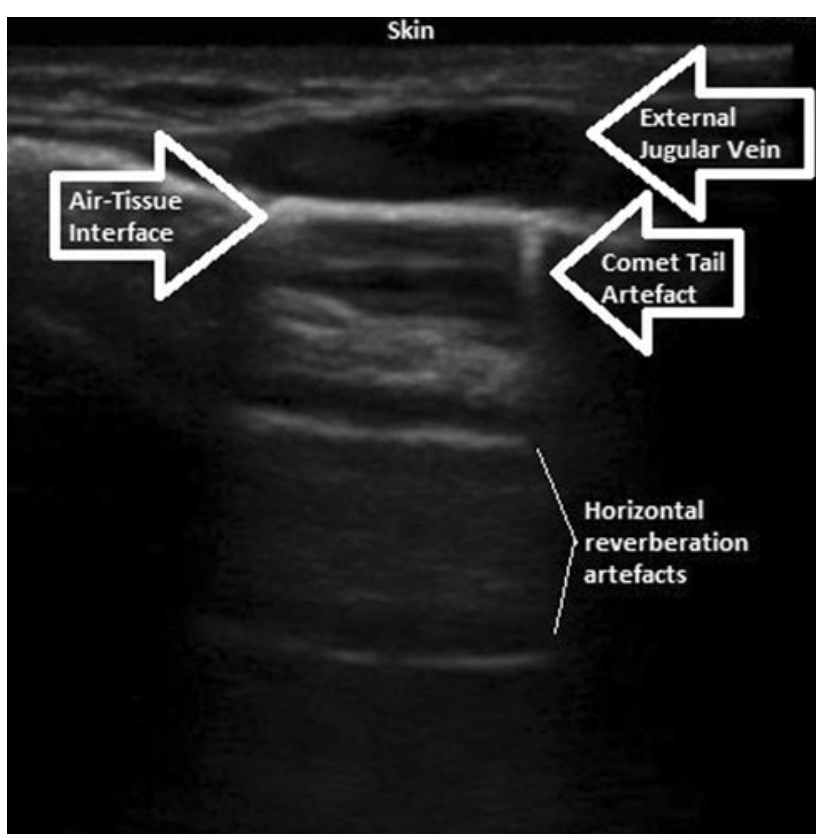

Fig. 1 Scan of the anterolateral R neck at the level of the cricoid cartilage. Just beneath the surface, the external jugular vein can be seen with a bright hyperechoic line beneath it representing the air in the tissue planes. Two horizontal reverberation artifacts are beneath this line, and one comet-tail artifact can be seen

equation $100(\mathrm{Z} 2-\mathrm{Z} 1)^{2} /(\mathrm{Z} 2+\mathrm{Z} 1)^{2}$, where $\mathrm{Z} 1$ and $\mathrm{Z} 2$ are the acoustic impedances of medium 1 and 2, respectively.

Due to the large difference in acoustic impedance between air (approx. 0.0004 Mrayls) and soft tissue (approx. 1.6 Mrayls), most of the ultrasound is reflected. Essentially, the tissue-air interface acts as a barrier to ultrasound, and only artifacts can be visualized deep within the structure. Comet tail artifacts (Fig. 1) also indicate an interface between media with marked differences in acoustic impedance just distal to a strongly reflecting 


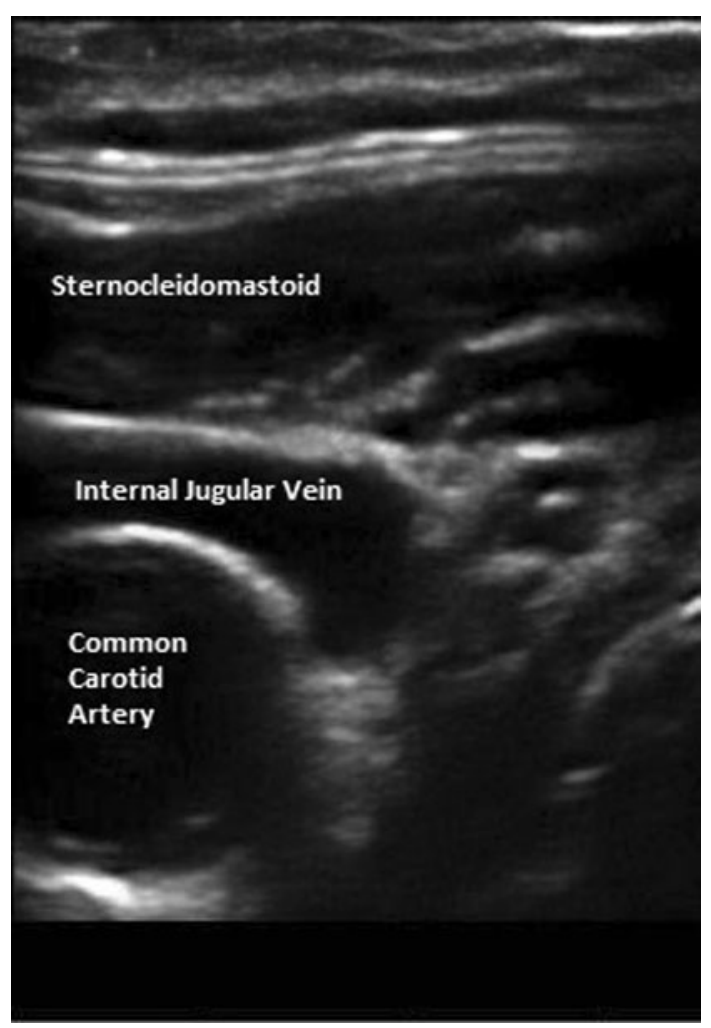

Fig. 2 Scan of the anterolateral $\mathrm{R}$ neck 13 days later, at a similar level as in Fig. 1. The sternocleidomastoid muscle is identified easily, and beneath is the internal jugular vein above the carotid artery structure. ${ }^{2}$ This case clearly shows that air in the subcutaneous tissue will limit the utility of ultrasound in internal jugular vein cannulation. The air makes it impossible to localize the vessels underneath; instead, many different artifacts are shown.

Disclaimer No funding was received for this article from any source, and there is no commercial or non-commercial affiliation.

Competing interests None declared.

\section{References}

1. Neustein $S$. Mandating ultrasound usage for internal jugular vein cannulation. Can J Anesth 2010; 57: 868-9.

2. Thickman DI, Ziskin MC, Goldenberg NJ, Linder BE. Clinical manifestations of the comet tail artifact. J Ultrasound Med 1983; 2: $225-30$ 